\title{
Higher Red Blood Cell Distribution Width is a Poor Prognostic Factor for Patients with Chronic Myeloid Leukemia
}

This article was published in the following Dove Press journal: Cancer Management and Research

\author{
Tao $\mathrm{Li}^{1,2}$ \\ Xin $\mathrm{Li}^{1}$ \\ Hui Chen ${ }^{3}$ \\ Kai-Zhao Huang' \\ Qi Xie ${ }^{1,2}$ \\ Han-Yu Ge' \\ Shen-Meng $\mathrm{Gao}^{4}$ \\ Jian-Hua Feng $\mathbb{B D}^{4}$ \\ Jun-Jun Yang' \\ Zhan-Guo Chen' \\ Xiao-Qun Zheng $\mathbb{I D}^{1,2}$ \\ 'Department of Laboratory Medicine, \\ The Second Affiliated Hospital and Yuying \\ Children's Hospital of Wenzhou Medical \\ University, Wenzhou, Zhejiang, People's \\ Republic of China; ${ }^{2}$ School of Laboratory \\ Medicine and Life Sciences, Wenzhou \\ Medical University, The Key Laboratory \\ of Laboratory Medicine, Ministry of \\ Education of China, Wenzhou, Zhejiang, \\ People's Republic of China; ${ }^{3}$ Department \\ of Emergency, The First Affiliated \\ Hospital of Wenzhou Medical University, \\ Wenzhou, Zhejiang, People's Republic of \\ China; ${ }^{4}$ Department of Hematology, The \\ First Affiliated Hospital of Wenzhou \\ Medical University, Wenzhou, Zhejiang, \\ People's Republic of China
}

Correspondence: Zhan-Guo Chen; Xiao-Qun Zheng

Department of Laboratory Medicine, The Second Affiliated Hospital and Yuying Children's Hospital of Wenzhou Medical University, Wenzhou, Zhejiang, People's Republic of China

Tel +86-I3968806755; +86-13906653839

Email steve0577@I26.com;

jszhengxq@I63.com
Purpose: Red blood cell distribution width (RDW) has been considered as a potential indicator of the effects of treatment or as a prognostic indicator for various malignancies. Most chronic myeloid leukemia (CML) patients are in the chronic phase, but some have transformed to accelerated phase or blast phase (blast crisis). However, the clinical significance of RDW in CML remains limited.

Patients and Methods: In the present study, detailed clinical information and the RDW of 168 healthy people and $153 \mathrm{CML}$ patients (106 patients for the training cohort and 47 patients for the validation cohort) were retrospectively assessed.

Results: Multivariate analysis demonstrated that patient age (OR, $1.081 ; 95 \mathrm{CI} \%$ $1.039 \sim 1.125 ; p<0.001)$, platelet counts (OR, 0.997; 95CI\% 0.994 0.999; $p=0.001$ ) and RDW at admission (OR, 1.469; 95CI\% 1.121 1.925; $p=0.005)$ were significantly associated with the patients with advanced phase. Among CML patients in the chronic phase, higher RDW was significantly associated with overall survival (OS; $p=0.0008)$ and the event-free survival (EFS; $p=0.0221$ ) among CML patients with chronic phase, but not with Transformation-free survival (TFS; $p=0.0821$ ). Furthermore, higher RDW was associated with higher mortality compared to patients with low RDW (CML-associated deaths; $p<$ 0.0001). In addition, a decline in RDW is associated with the treatment of CML patients with tyrosine kinase inhibitors, especially at 6 and 12 months after the start of treatment.

Conclusion: Higher RDW is a potential prognostic biomarker for chronic CML patients.

Keywords: red blood cell distribution width, chronic myeloid leukemia, chronic phase, adverse prognosis

\section{Introduction}

Chronic myeloid leukemia (CML) is a myeloproliferative disorder characterized by a reciprocal chromosomal translocation which forms the abnormal BCR-ABL1 fusion gene because of an acquired reciprocal $t(9 ; 22)$ (q34; q11) translocation. The disease is traditionally described as manifesting in one of the three distinct clinical phases: chronic phase (CP), accelerated phase (AP), and blast crisis (BC). ${ }^{1}$ The introduction of tyrosine kinase inhibitors (TKIs) dramatically improved the outcomes of patients with CML. At present, patients with chronic phase CML (CML-CP) can attain long-term survival. $^{2,3}$ The Sokal, Hasford, and European Treatment and Outcome Study (EUTOS) scores are used to determine the prognosis of CML patients. However, there are still some patients in which the treatment failed and their 
CML-CP transformed to CML-AP or CML-BC. ${ }^{4-6}$ Hoffmann VS et al's team reported that $3.1-14 \%$ of CML patients had progressive clinical features at diagnosis or during long-term follow-up. ${ }^{7}$ In previous studies, clinical workers usually focused on the effect of treating CML-CP patients with TKIs. ${ }^{8,9}$ Although the prognosis of patients with CML-CP is accurate, the characteristics and markers for determining the prognosis of the advanced phase of CML are still not clear. The BCR-ABL1 transcript and somatic variants in epigenetic modifiers were confirmed, and these can be used to predict the response to imatinib. ${ }^{10,11}$ Lauseker $M$ et al's study determined that blast counts, age, chromosomal aberrations, and hemoglobin are the prognostic factors for patients with advanced phase. ${ }^{12}$ In another study, the results showed that many factors such as myeloid immunophenotype, treatment with TKIs before transformation to CML-BC, patients' age, and platelet counts $<102 \times 10^{9} / \mathrm{L}$ could predict poor survival rates in CML-BC patients. ${ }^{13}$ Studies have discovered various prognostic factors that are related to chronic or advanced phase of CML. However, for patients diagnosed with CML the factors that can be used to determine their prognosis and survival outcomes are still not clear.

During follow-up periods, a complete Blood Count (CBC) is used to assess the effect of treating CML patients with TKIs. ${ }^{14}$ The red blood cell distribution width (RDW) is a simple parameter that is part of the standard full blood count and measures of the heterogeneity in the size of a circulating patient's erythrocytes. The RDW is provided by automated hematology analyzers and it reflects the distribution of the red blood cell (RBC) volume. ${ }^{15}$ There is increasing evidence that an elevated RDW is associated with a poor prognosis for people in the general population, ${ }^{16,17}$ as well as in patients with coronary artery disease, ${ }^{18-20}$ metabolic syndrome, $^{21}$ and heart failure. ${ }^{22,23}$ Traditionally, RDW has been used in the investigation of the etiology of anemia. $^{24}$ The previous study only used the upper limit of the normal RDW range (RDW value $15.0 \%$ ) to predict the prognosis and the response of CML-CP patients to treatment. ${ }^{25}$ However, it may not be suitable for determining the prognosis of CML patients when they are diagnosed at their first admission. Yuta Baba et al's team identified a new RDW cutoff using different clinical outcomes of patients with myelodysplastic syndrome. $^{26}$ Therefore, the hypothesis that different
RDW values indicate the occurrence and the different phases of CML is proposed. In addition, it is suggested that assessing patients' RDW is more favorable for assessing their treatment and for their follow-up evaluations.

The present study found that RDW was temporarily elevated when the CML-CP of patients transformed to the advance phase. The present study used X-tile software and data from a training and validation cohort established the most suitable RDW cutoff value based on the different phases of CML. In addition, univariate and multivariate analyses were used to determine which factors are associated with the advanced phase of CML in all patients. Based on the records of the patients, the role of RDW as a predictor of the prognosis and survival outcomes of the patients was also confirmed in the present study.

\section{Patients and Methods}

\section{Patients}

Our study retrospectively reviewed $153 \mathrm{CML}$ patients from January 2009 to December 2019 at the Second Affiliated Hospital \& Yuying Children's Hospital of Wenzhou Medical University. The patients were all over 18 years of age and were treated with TKIs and their outcome was followed up for at least 12 months. CML$\mathrm{CP}$ and CML-AP/BC were diagnosed according to the European Leukemia Net (ELN2013) criteria. $^{27}$

The criteria for excluding patients from the present study were: patient's age $<18$ years $(n=19)$; patient's data were not completed $(\mathrm{n}=11)$; patients without followup data $(\mathrm{n}=7)$; patients initially treated with interferon- $\alpha$ $(\mathrm{n}=8)$; patients treated with any chemotherapeutic agent prior to or in combination with TKI treatment $(n=6)$; patients that received a blood transfusion prior to TKI treatment $(\mathrm{n}=9)$; patients that received a hematopoietic stem cell transplant during follow-up $(n=8)$; and patients who had an NYHA grade of III/IV $(n=3)$. The framework and criteria used to select patients for inclusion in the present study are described in Figure 1.

The clinical characteristics of the patients that were recorded included their age, gender, history of smoking, Splenomegaly, history of cardiovascular system diseases, and with what they were initially treated. We defined a patient as a nonsmoker if they had never smoked or had smoked less than 100 cigarettes in their lifetime. All others were classified as smokers. The detailed clinical information of the patients is 
Patients were diagnosed as chronic myeloid leukemia (CML) from January 2009 to

December 2019 at The Second Affiliated Hospital \&Yuying Children's Hospital of Wenzhou Medical University $(n=224)$

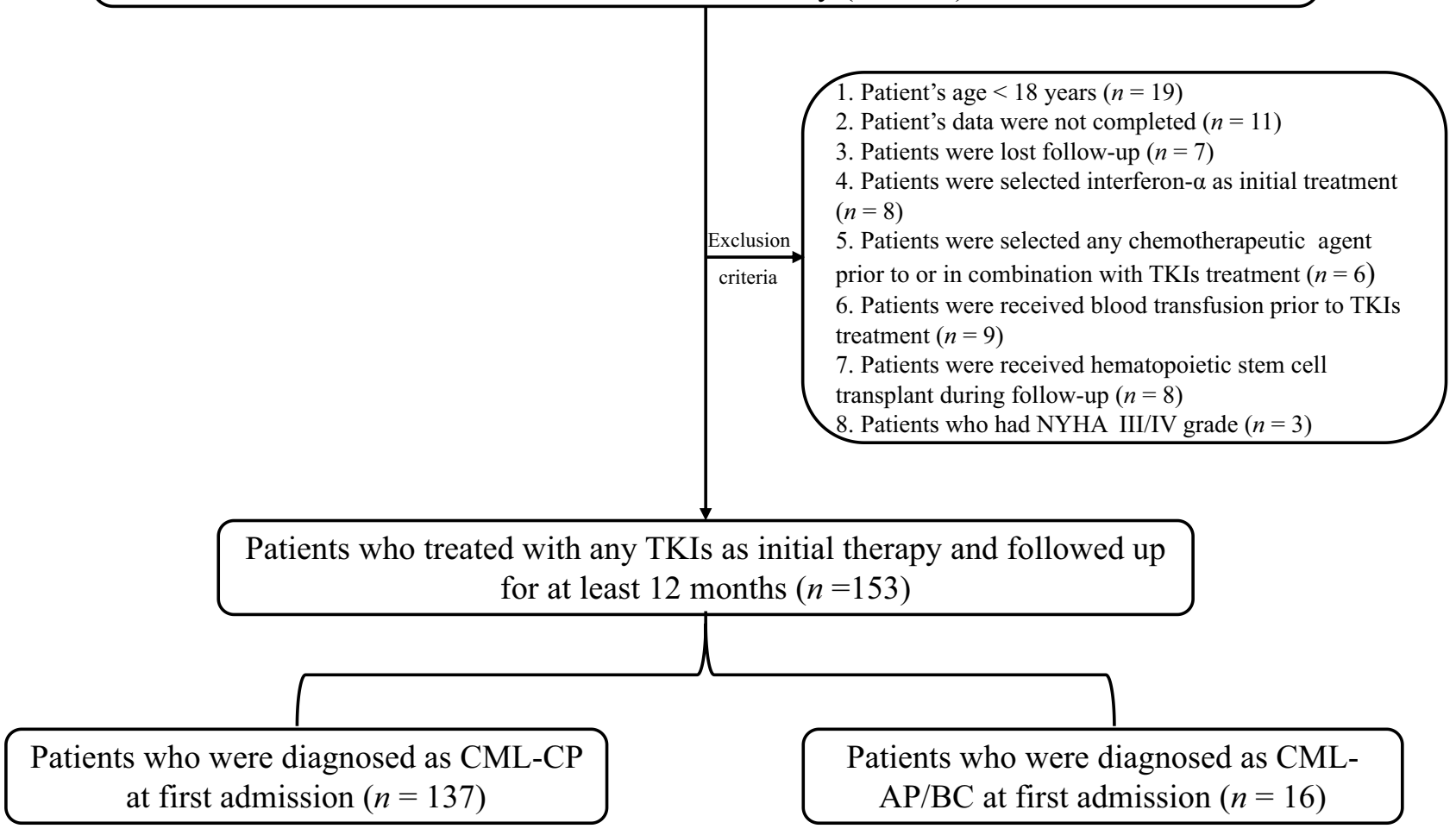

Figure I Flow chart of the criteria used to select the patients for inclusion in the present study.

summarized in Table 1. The definitions of a partial cytogenetic response (PCyR), a major molecular response (MMR), and a complete cytogenetic response (CCyR) were in accordance with the recommendations of the ELN 2013. The categories of response to treatment with TKIs included optimal, warning, and failure responses, and were defined according to the ELN 2013 recommendations. ${ }^{27}$

The overall survival (OS) of a patient was defined as the length of time from diagnosis to death or the last follow-up. Event-free survival (EFS) was defined as the time period from the date of diagnosis to the date of the first event or the last follow-up. Events were defined as either two consecutive confirmations of a loss of CCyR, the transformation of CML-CP to CML-AP/BC, or any cause of death. Transformation-free survival (TFS) was defined as the period from the date of the diagnosis when the patient's CML-CP transformed to CML-AP/BC or the last follow-up. All of the patients consented to participate in the present study by signing an informed consent form. The present study was approved by the Research Ethics Board of The Second Affiliated Hospital \& Yuying
Children's Hospital of Wenzhou Medical University (Number: LCKY2020-430) and it was conducted in accordance with the Declaration of Helsinki.

\section{Complete Blood Count (CBC) and RDW Values}

In our study, the $\mathrm{CBC}$ was obtained from patients' peripheral venous blood using a Sysmex XI-5000, Japan. The RDW values at diagnosis were obtained prior to treatment. Accordingly, the range $11.6 \sim 15.0 \%$ was considered normal.

To avoid overfitting and to analyze the interobserver reproducibility of the RDW values, the CML patients were randomly assigned to training cohort $(\mathrm{n}=106 ; 70 \%)$ and validation cohort $(\mathrm{n}=47 ; 30 \%)$ using $\mathrm{R}$ software version 3.0.1 (http://www.R-project.org). The distribution of the $p$ value of the RDW cutoff value was determined using $\mathrm{X}$-tile software, version 3.6.1 (Yale University School of Medicine, New Haven, Conn) and also confirmed using the training cohort, which included patients with advanced phase. 


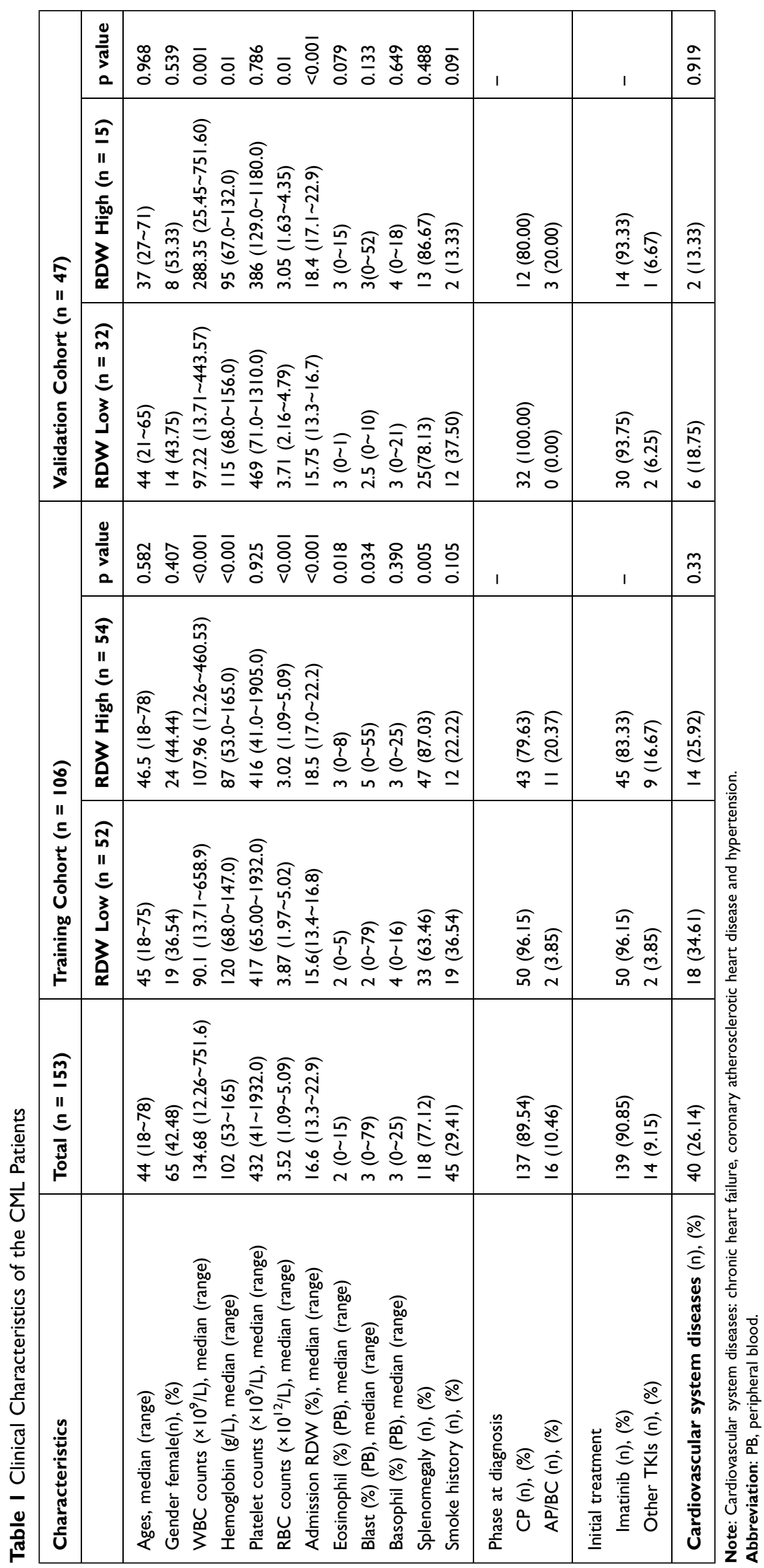




\section{Statistical Analysis}

The statistical analyses were performed using the software SPSS V. 26.0 (SPSS; Chicago, IL, USA). Categorical data were compared between groups using Fisher's exact test or the chi-square test. Continuous data were compared using the $t$-test. The relationship between the clinical features and the advanced phase of CML were analyzed using univariate and multivariate analyses. The Kaplan-Meier method was used to analyze OS, EFS, TFS, and the CMLassociated deaths. The Log rank test was used to compare OS, EFS, TFS, and CML-associated deaths between groups of patients with low and high RDW values. A $p$ value less than 0.05 was considered statistically significant.

\section{Results}

\section{Clinical Characteristics of the CML Patients}

The 153 CML patients included in the present study consisted of 137 and 16 newly diagnosed CML-CP and CML-AP/BC patients, respectively. During follow-up examinations, the CML-CP of 11 patients was determined to have transformed to CML-AP/BC. One-hundred and eighteen of the 137 patients with CML-CP had RDW values higher (86.13\%; range 13.3 22.9\%) than the normal range. The 16 patients diagnosed with CML-AP/BC when diagnosed all had RDW values (100\%; range 15.2 19.9\%) that were higher than the normal range.

The patients in the high RDW group had higher WBC counts $(p<0.001)$, lower hemoglobin levels $(p<0.001)$, lower RBC counts $(p<0.001)$, higher RDW when diagnosed $(p<0.001)$, a higher probability of splenomegaly, and higher probabilities of blast and eosinophils in the peripheral blood $(p<0.05)$ compared to patients in the low RDW group and in the training cohort, whereas, when comparing patients in the high and low RDW groups, no differences were observed in their ages, gender, platelet count, history of smoking, or diseases of their cardiovascular systems (Table 1). Furthermore, based on patients in the validation cohort, differences in the WBC count, hemoglobin level, RBC count, and RDW when diagnosed were also found for patients in the low and high RDW groups in the validation cohort $(p<0.05)$. No statistical differences were observed in any of the other clinical features (Table 1). Our study also additionally analyzed the differences in patients with CP between low and high RDW groups in patients with $\mathrm{CP}$ (Supplement Table 1).

\section{The Most Suitable RDW Cutoff Value Related to the Advanced Phase (CML-AP/ $\mathrm{BC})$}

There were 137 newly diagnosed CML-CP patients, 16 newly diagnosed CML-AP/BC patients, and 11 patients developed to CML-AP/BC during the followed period. Besides, our study also collected 168 healthy people's RDW value, who did not have tumors and other diseases (age; range 18 68 years). There were significant differences in RDW value among Normal and CML-CP groups ( $p<0.001$; Figure 1A), Normal and CML-AP/BC groups ( $p<0.0001$; Figure $2 \mathrm{~A}$ ), CML-CP and CML-AP/BC groups ( $p=0.0003$; Figure 2A).

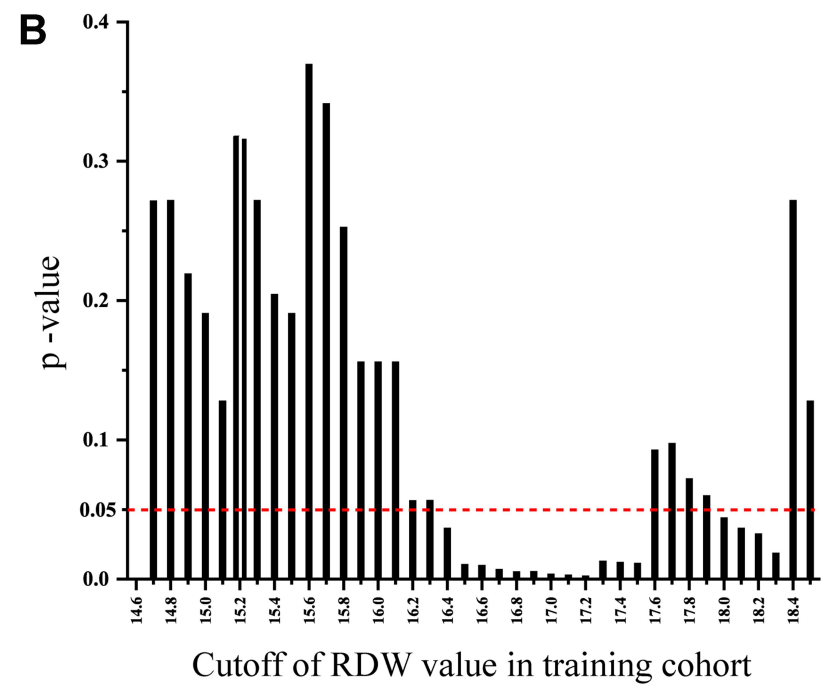

Figure 2 (A) Differences in the RDW values between healthy people (normal, $n=168$ ) and patients with CML-CP $(n=137)$ and CML-AP/BC $(n=27)$. $(\mathbf{B})$ The distribution of the $p$ values of the different RDW cutoff values of the patients $(n=106)$ in the training cohort $(* * * 00.001)$. 


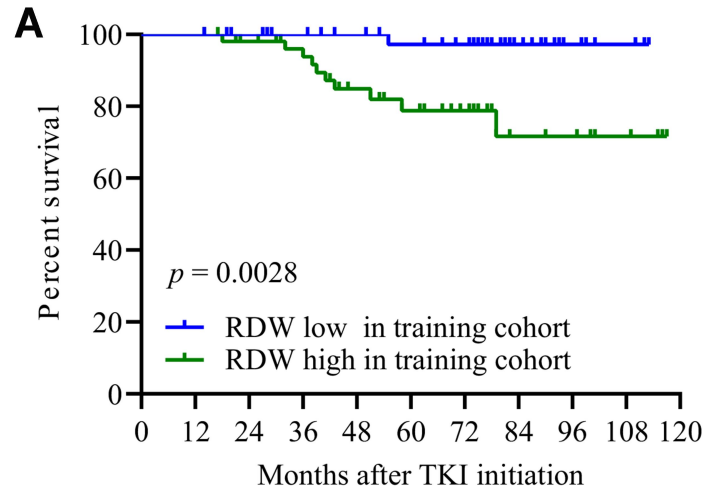

Number of patients

$\begin{array}{llllllllllll}\text { Low } & 52 & 52 & 48 & 45 & 40 & 34 & 25 & 13 & 7 & 3 & 0\end{array}$

$\begin{array}{llllllllllll}\text { High } & 54 & 54 & 49 & 44 & 29 & 24 & 16 & 8 & 7 & 4 & 0\end{array}$

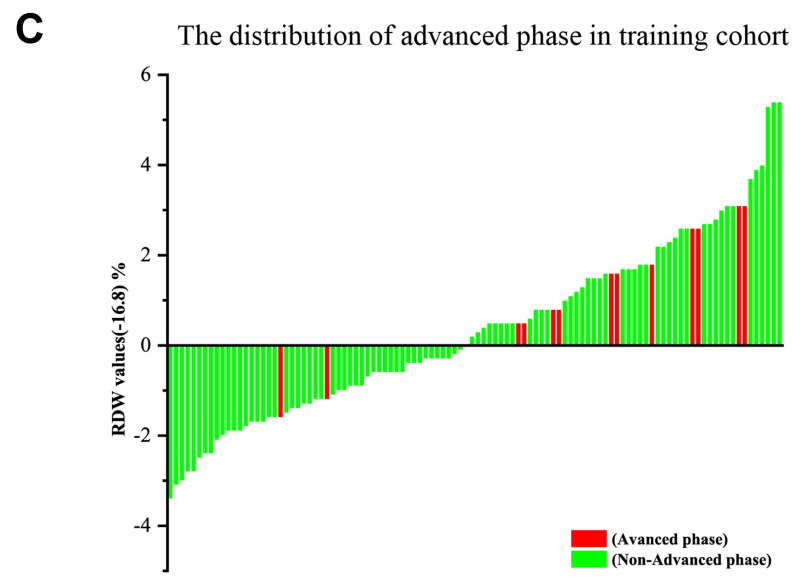

B

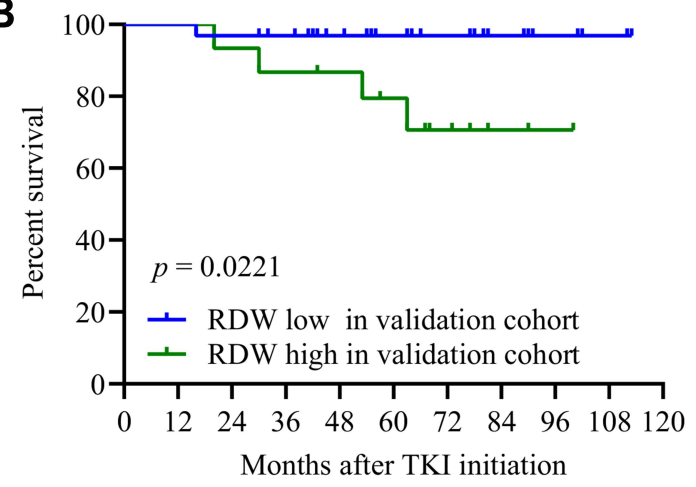

Number of patients

$\begin{array}{llllllllllll}\text { Low } & 32 & 32 & 31 & 29 & 24 & 20 & 16 & 9 & 6 & 4 & 0\end{array}$

$\begin{array}{lllllllllll}\text { High } & 15 & 15 & 14 & 13 & 12 & 9 & 6 & 3 & 1 & 0\end{array}$

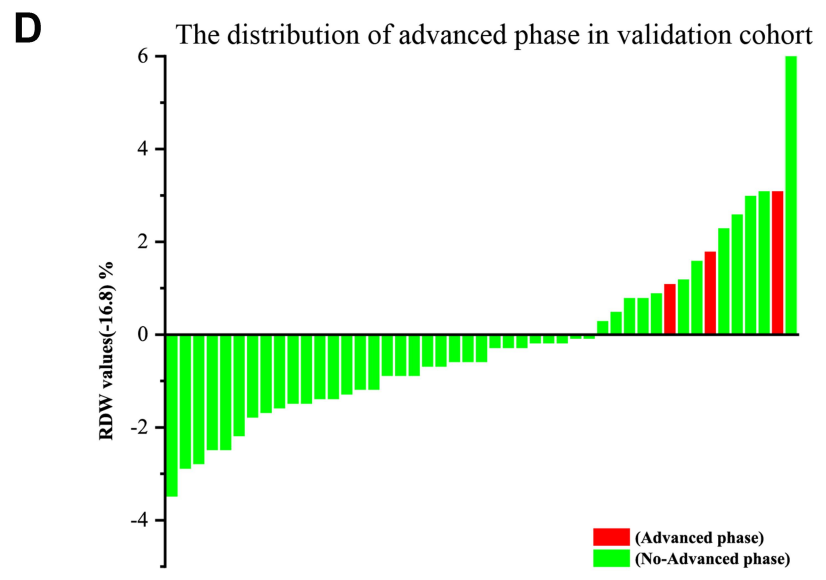

Figure 3 The overall survival of patients in the training $(\mathbf{A})$ and validation $(\mathbf{B})$ cohorts. The distribution of patients in the training $(\mathbf{C})$ and validation (D) cohorts with advanced phase CML and in the high and low RDW groups which were based on the RDW cutoff value.

Application of the X-tile software to the training cohort (n $=106,90 \%$ ) determined that an RDW value of $16.8 \%$ distinguished between patients with CML-CP and CML-AP/BC $(p=0.0069$, Figure 2B). Application of the Kaplan-Meier method to the training ( $p=0.0028$, Figure $3 \mathrm{~A}$ ) and validation cohort ( $p=0.0221$, Figure $3 \mathrm{~B}$ ) showed that patients in both cohorts with higher RDW values have significantly shorter OS than patients with low RDW values. Furthermore, the distribution of patients in the training (Figure 3C) and validation (Figure 3C) cohorts with advanced phase CML in the high and low RDW groups are also shown.

To further prove the practicality of using this RDW cutoff value to predict the CML phase, Spearman's rank correlation coefficient was used to determine if there is a correlation between RDW values and blast cells in the bone marrow when diagnosed. The result showed that the RDW value is a better factor for predicting patients with the advanced phase $(p=0.011 ; r=0.543)$ than patients with CML-CP.

\section{The RDW Value as a Predictor of} Patients' Response to Treatment and the
Prognosis of Patients with CML-CP Our study investigated the extent to which the RDW value could predict CML patients' response to treatment as measured using the recommendations of the ELN 2013. Compared to the CML patients in the low RDW group, the CML patients in the high RDW group were associated with a significantly poorer response to treatment after 3,6 , and 12 months after the commencement of treatment (Table 2).

The OS ( $p=0.0008$; Figure 4A) and EFS ( $p=0.0221$; Figure 4B) of patients in the high RDW group were significantly less than that of the patients in the low RWS group, respectively. Furthermore, the CML-associated deaths were significantly higher in patients with advanced CML (83.3\%) compared to patients with CML-CP and with high (14.5\%) and low $(2.4 \%)$ RDW values $(p<0.001$; Figure 4D). The RDW 
Table 2 Associations Between RDW and the Responses Treatment with TKIs

\begin{tabular}{|l|l|l|l|}
\hline & $\begin{array}{l}3 \text { Months Optimal/Warning/ } \\
\text { Failure }\end{array}$ & $\begin{array}{l}\text { 6 Months Optimal/Warning/ } \\
\text { Failure }\end{array}$ & $\begin{array}{l}\text { I2 Months Optimal/Warning/ } \\
\text { Failure }\end{array}$ \\
\hline RDW Low $(\mathrm{n}=81)$ & $77 / 3 / 1$ & $73 / 5 / 3$ & $72 / 7 / 2$ \\
RDW High $(\mathrm{n}=56)$ & $45 / 7 / 4$ & $37 / 10 / 9$ & $31 / 12 / 13$ \\
p value & 0.024 & 0.002 & $<0.001$ \\
\hline
\end{tabular}

values could not be used to predict TFS ( $p=0.0821$; Figure 4C).

\section{Changes in the RDW Values of CML Patients That Were Treated Over Time with TKIs}

To evaluate the correlation between the RDW values and the therapeutic effect of TKIs, the dynamic changes in the RDW values of CML patients following treatment over time with TKIs were analyzed. The RDW value was initially elevated at 1 month (median $16.6 \%$ when diagnosed and $17.6 \%$ after 1 month of treatment; $p=0.001$, Figure 5A) but declined significantly after 3 months (median $15.7 \%$ at 3 months; $p=0.001$ Figure $4 \mathrm{~A}$ ) of treatment with TKIs. Furthermore, compared with the RDW values of the CML patients at diagnosis, the RDW values were significantly lower after 6 months of treatment with TKIs treatment (median14.5\%; $p<0.001$, Figure 5A).

To compare the longitudinal changes of the RDW values between patients in the low and high RDW groups, the patients with CML-CP were compared with patients with CML-CP and advanced CML, ie, all-CML. The RDW values of the patients in all CML and low RDW groups at diagnosis returned to the normal range after 6 months of treatment with TKIs (Figure 5B).It took 2 years for the patients in, while the high RDW group patients tend to return to the normal range in 2 years (Figure $5 \mathrm{~B}$ ).

\section{Factors Associated with Advanced Phase CML (CML-AP/BC) in All CML Patients: Univariate and Multivariate Analyses}

The univariate analysis showed that ages (OR, 1.063; 95\% CI, 1.029 91.098; $p<0.001$ ), WBC counts (OR, 0.214; $p<0.001$ ), RBC count (OR, 0.594; 95\% CI, 0.356 0.992; $p<0.001$ ), hemoglobin level (OR, 0.970; 95\% CI, $0.952 \sim 0.989 ; p=0.047)$, RDW value when diagnosed (OR, 1.264; 95\% CI, 1.024 1.559; $p=0.029$ ), platelet count (OR, 0.997; 95\% CI, 0.996 0.999; $p=0.006$ ), eosinophil in PB (OR, 0.214; $p<0.001)$, basophil in PB
(OR, <0.001; 95\% CI, 1.029 91.098; $p<0.001$ ), history of cardiovascular system disease (OR, 1.648; 95\% CI, $1.103 \sim 2.463 ; p=0.001)$ were all significantly associated with advanced phase (Table 3). However, gender, history of smoking, and splenomegaly were not associated with the advanced phase (Table 3 ).

The multivariate analysis showed that age (OR,1.081; 95CI\% 1.039 1.125; $p<0.001)$, being a female (OR,0.332; 95CI\% 0.119 0.926; $p=0.035$ ), platelet count (OR,0.997; 95CI\% 0.994 0.999; $p=0.001$ ), RDW value when diagnosed (OR,1.469; 95CI\% 1.121 1.925; $p=0.005$ ) were correlated with advanced phase, but while not any of the other variables (Table 3 ).

\section{Discussion}

CML is a neoplastic disease, and its genetic and cytogenetic changes play important roles in the prognosis and treatment process of patients. Our study demonstrated that higher RDW values are an adverse prognostic and survival outcomes biomarker for CML-CP patients. The multivariate analysis demonstrated that age, platelet count, and RDW at diagnosis were related to the advanced phase. Consequently, the dynamic changes of the RDW values of CML patients could help clinical workers manage the follow-up treatment of the patients. In agreement with the significance of responses to early treatment, the RDW value at diagnosis also predicted the outcomes, because it reflected an early response at 3 months, to treatment. ${ }^{28}$ The vast majority of CML patients are diagnosed with CML-CP. However, some patients present with features of advanced phase at diagnosis. Most studies analyzing prognostic factors and survival outcomes for CML-AP/BC refer to patients that developed this advanced phase of CML from an initial diagnosis of CML-CP. ${ }^{29,30}$ The results of these studies indicate that despite the availability of TKIs, the treatment options and the outcome for these patients are still poor. After transformation from CML-CP to CML-BC, the median survival time in a German CML-study IV was 7.9 months. ${ }^{13}$ Therefore, there is still a need for more 


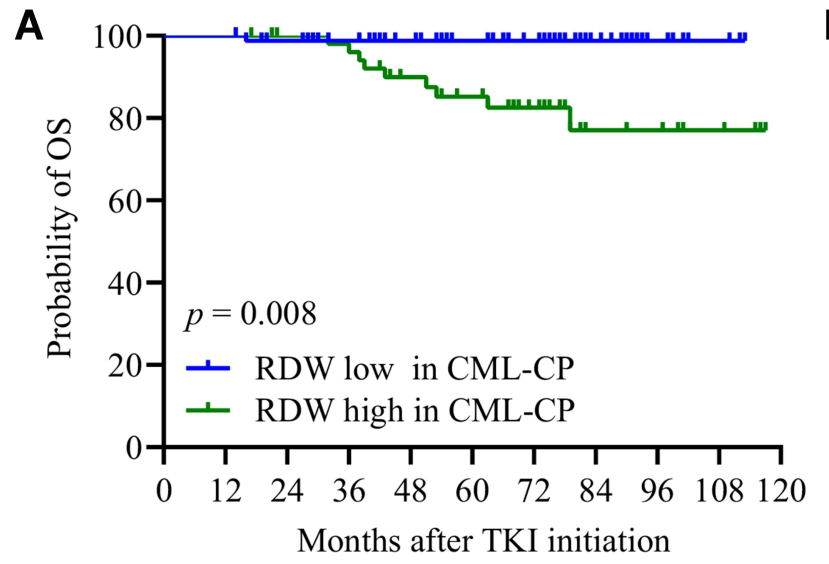

Number of patients

$\begin{array}{llllllllllll}\text { Low } & 82 & 82 & 77 & 72 & 63 & 50 & 46 & 25 & 13 & 7 & 0\end{array}$

$\begin{array}{llllllllllll}\text { High } & 55 & 55 & 51 & 49 & 38 & 33 & 22 & 11 & 8 & 4 & 0\end{array}$

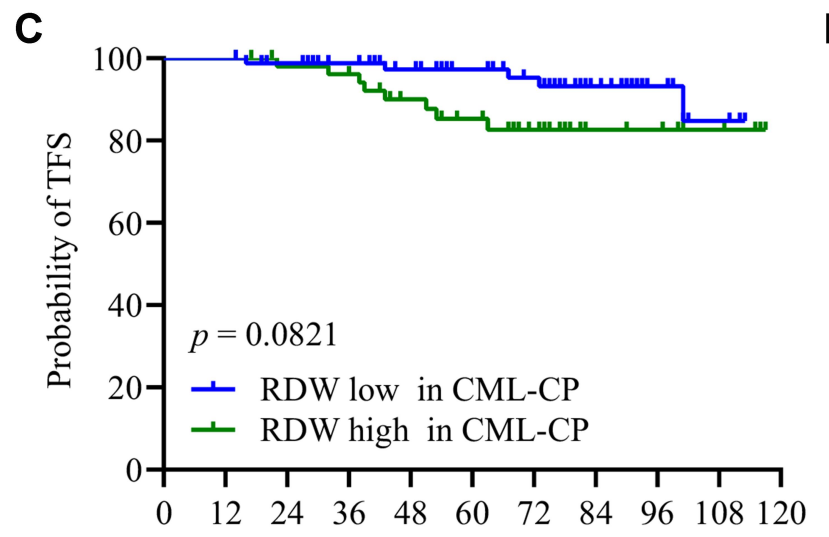

Months after TKI initiation

Number of patients

$\begin{array}{llllllllllll}\text { Low } & 82 & 82 & 77 & 72 & 63 & 50 & 46 & 25 & 13 & 7 & 0\end{array}$

$\begin{array}{llllllllllll}\text { High } & 55 & 55 & 51 & 49 & 38 & 33 & 22 & 11 & 8 & 4 & 0\end{array}$
B

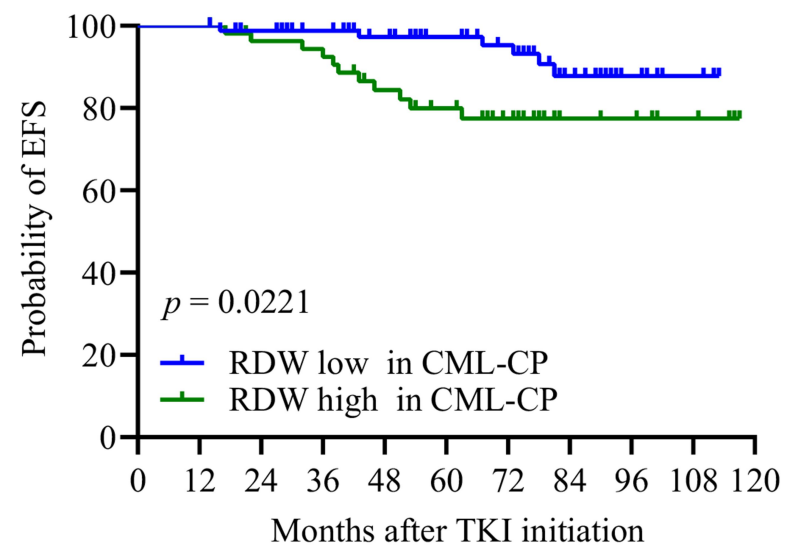

Number of patients

$\begin{array}{llllllllllll}\text { Low } & 82 & 82 & 77 & 72 & 63 & 50 & 46 & 25 & 13 & 7 & 0\end{array}$

$\begin{array}{lllllllllllll}\text { High } & 55 & 55 & 51 & 49 & 38 & 33 & 22 & 11 & 8 & 4 & 0\end{array}$

D

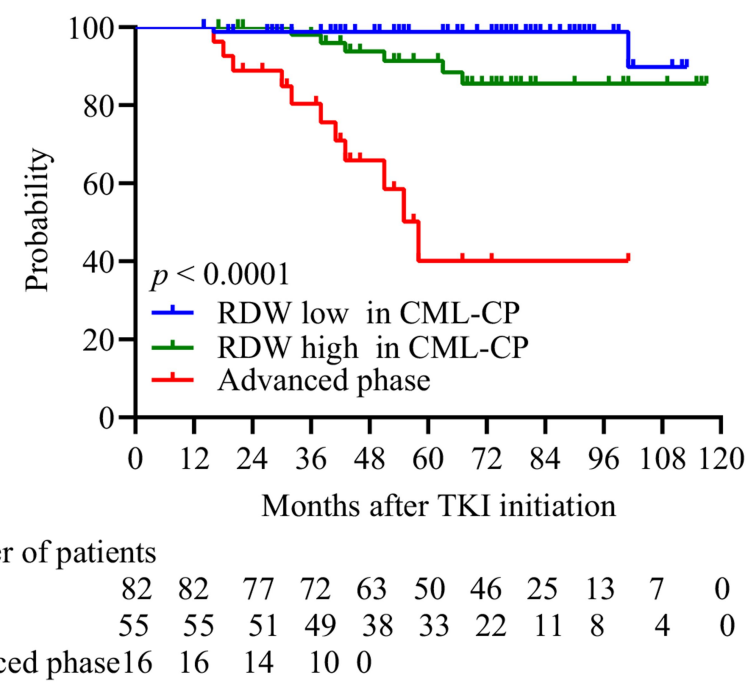

Figure 4 The relationship between CML-CP patients with low and high RDW values and their (A) OS, (B) EFS, and (C) TFS. (D) The death rates of patients with CML-CP in the low and high RDW groups, and those with advanced phase CML, respectively.

markers to predict the advanced phase and for the prognosing patients with CML-CP. Detection of the BCRABL1 transcript level is the gold standard method for monitoring CML minimal residual disease and the optimal management of CML patients. ${ }^{31}$ Although there are many emerging technologies, such as digital PCR, to detect the transcription level of BCR-ABL1, ${ }^{32-34}$ RDW detection is a widely available and inexpensive test that is performed as part of the complete blood count. It is important to point out that the average lifespan of RBCs is 120 days; thus, RDW could be used as an indicator during the long-term follow-up of patients with CML.
Our study showed that RDW plays an important role in determining the prognosis and the effect of treatment with TKIs. What follows is a description of the reasons why RDW plays a key prognostic role in CML. It is well established that the RDW value is elevated when the destruction of RBCs increases or there is an increase in the production of infective defective RBCs. ${ }^{15} \mathrm{CML}$ is a specific disease in which the $\mathrm{CML}$ stem cells have to differentiate towards the erythroid cell lineage, resulting in the involvement of malignant clone-derived erythropoiesis. ${ }^{35}$ Some studies have recently revealed that patients harboring IDH1/2 and ASXL1 mutations had significantly higher RDW values than those without these 


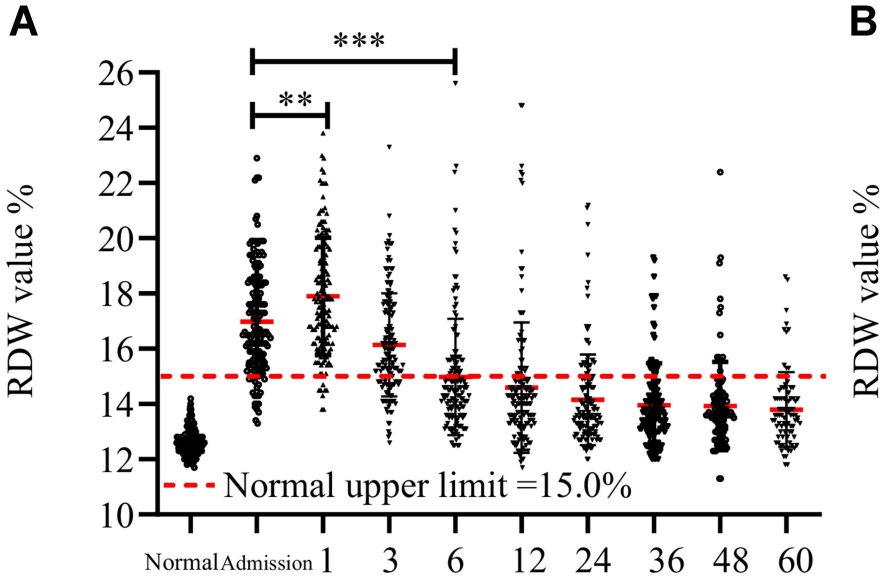

Months after TKI initiation
B

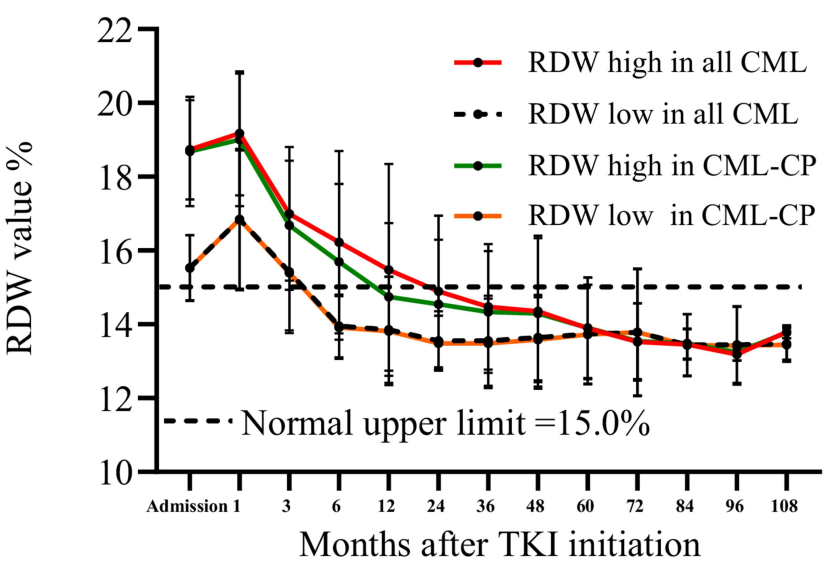

Figure 5 (A) Changes in the RDW value of patients with CML that were treated over time with TKIs. (B) Changes in the RDW values in the different groups of CML patients that were treated over time with TKIs. $\left({ }^{* *} p<0.01\right.$; $\left.* * * p<0.001\right)$.

mutations. ${ }^{36,37}$ Furthermore, these mutations are often present in CML cells and are associated with the status of the disease in CML patients. ${ }^{37}$ Although baseline prognostic factors for the efficacy of treatment with second TKIs have not yet been determined, the multiple predictors for the efficacy of treatment with imatinib have already been reported. A recent study showed that the Sokal low and medium/high groups responded equally to dasatinib treatment. ${ }^{38}$ Another study proved that Treatment-Free Remission after second-line nilotinib treatment in patients with CML-CP was greatly improved and none had CML progression to AP or BC. ${ }^{39}$ However, in our study, only 14 patients chose to be treated with the second TKIs treatment. We did not make a distinction between patients treated with first and second TKIs in our study, we analyzed the change in RDW values of patients treated with TKIs as a whole.

We were aware of some limitations in our study. Our study split the 153 patients into a training and validation cohort for the internal validation. However, the sample size of the CML patients that enrolled in the study was not large and the results of single-center retrospective cohort studies are not generalizable to other populations.

Table 3 Univariate and Multivariate Analyses of Factors for the Advanced Phase in All Patients

\begin{tabular}{|c|c|c|c|c|}
\hline Variables & Univariate Analysis OR $(95 \% \mathrm{Cl})$ & $p$ value & Multivariate Analysis OR $(95 \% \mathrm{Cl})$ & $p$ value \\
\hline Ages & $1.063(1.029 \sim 1.098)$ & $<0.001$ & 1.081 (I.039 1.125) & $<0.001$ \\
\hline Gender (Female) & $0.656(0.488 \sim 0.96 \mathrm{I})$ & 0.052 & $0.332(0.119 \sim 0.926)$ & 0.035 \\
\hline WBC counts $\left(\times 10^{9} / \mathrm{L}\right)$ & 0.214 & $<0.001$ & & \\
\hline RBC counts $\left(\times 10^{12} / \mathrm{L}\right)$ & $0.594(0.356 \sim 0.992)$ & 0.047 & & \\
\hline Hemoglobin $(g / L)$ & $0.970(0.952 \sim 0.989)$ & 0.002 & & \\
\hline RDW at diagnosis (\%) & $1.264(1.024 \sim 1.559)$ & 0.029 & $1.469(1.121 \sim 1.925)$ & 0.005 \\
\hline Platelet counts $\left(\times 10^{9} / \mathrm{L}\right)$ & $0.997(0.996 \sim 0.999)$ & 0.006 & $0.997(0.994 \sim 0.999)$ & 0.001 \\
\hline Eosinophil (\%; PB) & 0.214 & $<0.001$ & & \\
\hline Basophil (\%; PB) & $<0.001 \quad(0.001 \sim 0.003)$ & 0.011 & & \\
\hline \multicolumn{5}{|l|}{ Smoking status } \\
\hline Never-smoker & Reference & & & \\
\hline Ever-smoker & $0.99 \mid(0.522 \sim 1.882)$ & 0.978 & & \\
\hline Splenomegaly & $0.935(0.762 \sim 1.147)$ & 0.553 & & \\
\hline Cardiovascular system disease & $1.648(1.103 \sim 2.463)$ & 0.001 & & \\
\hline
\end{tabular}

Note: Cardiovascular system diseases: chronic heart failure, coronary atherosclerotic heart disease and hypertension. 


\section{Conclusion}

The stratification of CML patients according to their RDW value can be used to determine their prognosis, survival outcomes, and advanced phase. This stratification is beneficial to subsequent treatment. To avoid CML-CP transforming to advanced phase, patients whose $\mathrm{RDW} \geq 16.8 \%$ when diagnosed need more time for follow-up. In the future, we will validate these findings in a larger population, which may provide new insights into CML therapy and follow-up treatment.

\section{Funding}

This work was supported by the Natural Science Foundation of Zhejiang Province, China [No. LY18H200006], Science and Technology Planning Project of Wenzhou, Zhejiang, China (No. Y20180108), Basic Public Welfare Technology Research Project of Zhejiang Province [LGF20H200005], and the Lin He's New Medicine and Clinical Translation Academician Workstation Research Fund [18331203].

\section{Disclosure}

The authors report no conflicts of interest in this work.

\section{References}

1. Steegmann JL, Baccarani M, Breccia M, et al. European LeukemiaNet recommendations for the management and avoidance of adverse events of treatment in chronic myeloid leukaemia. Leukemia. 2016;30(8):1648-1671. doi:10.1038/leu.2016.104

2. Chereda B, Melo JV. Natural course and biology of CML. Ann Hematol. 2015;94(Suppl 2):S107-S121. doi:10.1007/s00277-015-2325-Z

3. Kantarjian H, O'Brien S, Jabbour E, et al. Impact of treatment end point definitions on perceived differences in long-term outcome with tyrosine kinase inhibitor therapy in chronic myeloid leukemia. $J$ Clin Oncol. 2011;29(23):3173-3178. doi:10.1200/JCO.2010.33.4169

4. Jabbour E, Cortes J, Nazha A, et al. EUTOS score is not predictive for survival and outcome in patients with early chronic phase chronic myeloid leukemia treated with tyrosine kinase inhibitors: a single institution experience. Blood. 2012;119(19):4524-4526. doi:10.1182/ blood-2011-10-388967

5. Tiribelli M, Bonifacio M, Calistri E, et al. EUTOS score predicts long-term outcome but not optimal response to imatinib in patients with chronic myeloid leukaemia. Leuk Res. 2013;37(11):1457-1460. doi:10.1016/j.leukres.2013.07.037

6. Yamamoto E, Fujisawa S, Hagihara M, et al. European treatment and outcome study score does not predict imatinib treatment response and outcome in chronic myeloid leukemia patients. Cancer Sci. 2014;105 (1):105-109. doi:10.1111/cas.12321

7. Hoffmann VS, Baccarani M, Hasford J, et al. Treatment and outcome of 2904 CML patients from the EUTOS population-based registry. Leukemia. 2017;31(3):593-601. doi:10.1038/leu.2016.246

8. Baccarani M, Druker BJ, Branford S, et al. Long-term response to imatinib is not affected by the initial dose in patients with Philadelphia chromosome-positive chronic myeloid leukemia in chronic phase: final update from the tyrosine kinase inhibitor optimization and selectivity (TOPS) study. Int J Hematol. 2014;99(5):616-624. doi:10.1007/ s12185-014-1566-2
9. Hehlmann R, Lauseker M, Saußele S, et al. Assessment of imatinib as first-line treatment of chronic myeloid leukemia: 10-year survival results of the randomized CML study IV and impact of non-CML determinants. Leukemia. 2017;31(11):2398-2406. doi:10.1038/ leu. 2017.253

10. Castagnetti F, Gugliotta G, Breccia M, et al. The BCR-ABL1 transcript type influences response and outcome in Philadelphia chromosome-positive chronic myeloid leukemia patients treated frontline with imatinib. Am J Hematol. 2017;92(8):797-805. doi:10.1002/ajh.24774

11. Nteliopoulos G, Bazeos A, Claudiani S, et al. Somatic variants in epigenetic modifiers can predict failure of response to imatinib but not to second-generation tyrosine kinase inhibitors. Haematologica. 2019;104(12):2400-2409. doi:10.3324/haematol.2018.200220

12. Lauseker M, Bachl K, Turkina A, et al. Prognosis of patients with chronic myeloid leukemia presenting in advanced phase is defined mainly by blast count, but also by age, chromosomal aberrations and hemoglobin. Am J Hematol. 2019;94(11):1236-1243. doi:10.1002/ajh.25628

13. Jain P, Kantarjian HM, Ghorab A, et al. Prognostic factors and survival outcomes in patients with chronic myeloid leukemia in blast phase in the tyrosine kinase inhibitor era: cohort study of 477 patients. Cancer. 2017;123(22):4391-4402. doi:10.1002/cncr.30864

14. Radich JP, Deininger M, Abboud CN, et al. Chronic myeloid leukemia, version 1.2019, NCCN clinical practice guidelines in oncology. $J$ Natl Compr Canc Netw. 2018;16(9):1108-1135. doi:10.6004/ jncen.2018.0071

15. Evans TC, Jehle D. The red blood cell distribution width. J Emerg Med. 1991;9(Suppl 1):71-74. doi:10.1016/0736-4679(91)90592-4

16. Patel KV, Semba RD, Ferrucci L, et al. Red cell distribution width and mortality in older adults: a meta-analysis. J Gerontol a Biol Sci Med Sci. 2010;65(3):258-265. doi:10.1093/gerona/glp163

17. Perlstein TS, Weuve J, Pfeffer MA, Beckman JA. Red blood cell distribution width and mortality risk in a community-based prospective cohort. Arch Intern Med. 2009;169(6):588-594. doi:10.1001/ archinternmed.2009.55

18. Cavusoglu E, Chopra V, Gupta A, et al. Relation between red blood cell distribution width (RDW) and all-cause mortality at two years in an unselected population referred for coronary angiography. Int J Cardiol. 2010;141(2):141-146. doi:10.1016/j.ijcard.2008.11.187

19. Tsuboi S, Miyauchi K, Kasai T, et al. Impact of red blood cell distribution width on long-term mortality in diabetic patients after percutaneous coronary intervention. Circ J. 2013;77(2):456-461. doi:10.1253/circj.cj-12-0730

20. Osadnik T, Strzelczyk J, Hawranek M, et al. Red cell distribution width is associated with long-term prognosis in patients with stable coronary artery disease. BMC Cardiovasc Disord. 2013;13(1):113. doi:10.1186/1471-2261-13-113

21. Sánchez-Chaparro MA, Calvo-Bonacho E, González-Quintela A, et al. Higher red blood cell distribution width is associated with the metabolic syndrome: results of the ibermutuamur cardiovascular risk assessment study. Diabetes Care. 2010;33(3):e40. doi:10.2337/dc09-1707

22. Felker GM, Allen LA, Pocock SJ, et al. Red cell distribution width as a novel prognostic marker in heart failure: data from the CHARM program and the duke databank. J Am Coll Cardiol. 2007;50 (1):40-47. doi:10.1016/j.jacc.2007.02.067

23. Huang YL, Hu ZD, Liu SJ, et al. Prognostic value of red blood cell distribution width for patients with heart failure: a systematic review and meta-analysis of cohort studies. PLoS One. 2014;9(8):e104861. doi:10.1371/journal.pone. 0104861

24. Bessman JD, Gilmer PR Jr, Gardner FH. Improved classification of anemias by MCV and RDW. Am J Clin Pathol. 1983;80(3):322-326. doi:10.1093/ajcp/80.3.322

25. Iriyama N, Hatta $\mathrm{Y}$, Kobayashi $\mathrm{S}$, et al. Higher red blood cell distribution width is an adverse prognostic factor in chronic-phase chronic myeloid leukemia patients treated with tyrosine kinase inhibitors. Anticancer Res. 2015;35(10):5473-5478. 
26. Baba Y, Saito B, Shimada S, et al. Association of red cell distribution width with clinical outcomes in myelodysplastic syndrome. Leuk Res. 2018;67:56-59. doi:10.1016/j.leukres.2018.02.004

27. Baccarani M, Deininger MW, Rosti G, et al. European LeukemiaNet recommendations for the management of chronic myeloid leukemia: 2013. Blood. 2013;122(6):872-884. doi:10.1182/blood-2013-05501569

28. Jabbour E, Kantarjian H. Chronic myeloid leukemia: 2020 update on diagnosis, therapy and monitoring. Am J Hematol. 2020;95 (6):691-709. doi:10.1002/ajh.25792

29. Hehlmann R, Saußele S, Voskanyan A, Silver RT. Management of CML-blast crisis. Best Pract Res Clin Haematol. 2016;29 (3):295-307. doi:10.1016/j.beha.2016.10.005

30. Jabbour EJ, Hughes TP, Cortés JE, Kantarjian HM, Hochhaus A. Potential mechanisms of disease progression and management of advanced-phase chronic myeloid leukemia. Leuk Lymphoma. 2014;55(7):1451-1462. doi:10.3109/10428194.2013.845883

31. Soverini S, De Benedittis C, Mancini M, Martinelli G. Best practices in chronic myeloid leukemia monitoring and management. Oncologist. 2016;21(5):626-633. doi:10.1634/theoncologist.20150337

32. Bernardi S, Malagola M, Zanaglio C, et al. Digital PCR improves the quantitation of DMR and the selection of CML candidates to TKIs discontinuation. Cancer Med. 2019;8(5):2041-2055. doi:10.1002/ cam4.2087

33. Nicolini FE, Dulucq S, Boureau L, et al. Evaluation of residual disease and TKI duration are critical predictive factors for molecular recurrence after stopping imatinib first-line in chronic phase CML patients. Clin Cancer Res. 2019;25(22):6606-6613. doi:10.1158/ 1078-0432.CCR-18-3373
34. Furuya D, Moriai M, Koizumi Y, et al. Analysis of major BCR-ABL1 mRNA by digital polymerase chain reaction is useful for prediction of international scale. Int $J$ Clin Oncol. 2019;24(7):871-875. doi:10.1007/s10147-019-01419-9

35. Dainiak N, Liu A, Dewey MC, Kulkarni V. Chromosome analysis of isolated colony erythroblasts in chronic myelogenous leukaemia. $\mathrm{Br}$ $J$ Haematol. 1984;56(3):507-512. doi:10.1111/j.1365-2141.1984. tb03980.x

36. Schmidt M, Rinke J, Schäfer V, et al. Molecular-defined clonal evolution in patients with chronic myeloid leukemia independent of the BCR-ABL status. Leukemia. 2014;28(12):2292-2299. doi:10.1038/leu.2014.272

37. Makishima H, Jankowska AM, McDevitt MA, et al. CBL, CBLB, TET2, ASXL1, and IDH1/2 mutations and additional chromosomal aberrations constitute molecular events in chronic myelogenous leukemia. Blood. 2011;117(21):e198-e206. doi:10.1182/blood-201006-292433

38. Iriyama N, Fujisawa S, Yoshida C, et al. Shorter halving time of BCR-ABL1 transcripts is a novel predictor for achievement of molecular responses in newly diagnosed chronic-phase chronic myeloid leukemia treated with dasatinib: results of the D-first study of Kanto CML study group. Am J Hematol. 2015;90(4):282-287. doi:10.1002/ ajh. 23923

39. Mahon FX, Boquimpani C, Kim DW, et al. Treatment-free remission after second-line nilotinib treatment in patients with chronic myeloid leukemia in chronic phase: results from a single-group, phase 2, open-label study. Ann Intern Med. 2018;168(7):461-470. doi:10.7326/M17-1094

\section{Publish your work in this journal}

Cancer Management and Research is an international, peer-reviewed open access journal focusing on cancer research and the optimal use of preventative and integrated treatment interventions to achieve improved outcomes, enhanced survival and quality of life for the cancer patient.
The manuscript management system is completely online and includes a very quick and fair peer-review system, which is all easy to use. Visit http://www.dovepress.com/testimonials.php to read real quotes from published authors. 